\title{
The black disk to be observed in the Orear region
}

\author{
I.M. Dremin
}

\author{
Lebedev Physical Institute, Moscow 119991, Russia
}

\begin{abstract}
It is argued that the very first signatures of the approach to the black disk asymptotical limit in hadron collisions may be observed in the differential cross section of elastic scattering. The exponentially decreasing with the angle (or $\sqrt{|t|}$ ) regime beyond the diffraction peak will become replaced by an oscillatory behavior or by the power-like falloff. Some estimates of energies where this can happen are presented.
\end{abstract}

PACS: $13.85 . \mathrm{Dz}$

Hadrons behave as extended objects in high-energy collisions. The extreme model widely discussed for many years is the asymptotical picture of two absolutely black Lorentz-contracted disks with complete absorption at any impact parameter. In the impact parameter formalism the total cross section is given by the expression

$$
\sigma_{t}(s)=2 \int d^{2} b\left(1-e^{-\Omega(s, \mathbf{b})}\right) .
$$

The integration is over the two-dimensional space of the impact parameter b. $\Omega(s, \mathbf{b})$ denotes the opaqueness (or blackness) of disks colliding with the c.m.s. energy $\sqrt{s}$ and the impact parameter $\mathbf{b}$. For absolutely black $(\Omega(s, \mathbf{b}) \rightarrow \infty, b \leq R ; \Omega(s, \mathbf{b})=0, b>R)$ and logarithmically expanding ( $R=R_{0} \ln s, R_{0}=$ const ) disks with radii $R$ one gets from (1)

$$
\sigma_{t}(s)=2 \pi R^{2}+O(\ln s) .
$$

The elastic and inelastic processes should contribute on equal footing

$$
\frac{\sigma_{e l}}{\sigma_{t}(s)}=\frac{\sigma_{i n}}{\sigma_{t}(s)}=\frac{1}{2} \mp O\left(\ln ^{-1} s\right) .
$$

The width of the diffraction peak $B^{-1}(s)$ should shrink because its slope increases as

$$
B(s)=\frac{R^{2}}{4}+O(\ln s) .
$$


The forward ratio of the real part to the imaginary part of the elastic scattering amplitude $\rho_{0}$ must vanish asymptotically as

$$
\rho_{0}(s)=\frac{\pi}{\ln s}+O\left(\ln ^{-2} s\right) .
$$

None of these asymptotical predictions were yet observed in experiment.

Total cross sections increase with energy albeit all fits taking into account the latest LHC data [1] prefer the power-like behavior. The preasymptotic origin may be ascribed to this effect, especially in view of some theoretical guesses [2] proposed long time ago.

Inelastic processes are about 3 to 4 times more abundant than elastic ones. Even though the elastic cross section increased from $18 \%$ of the total cross section at ISR energies to $25 \%$ at LHC energies, it is much less than $50 \%$ shown above.

The diffraction peak shrinks about twice from energies about $\sqrt{s} \approx 6$ $\mathrm{GeV}$ where $B \approx 10 \mathrm{GeV}^{-2}$ to the LHC energy where $B \approx 20 \mathrm{GeV}^{-2}$. At ISR energies the slope $B(s)$ increased logarithmically. To account for LHC data one needs a stronger than simple logarithmical dependence. The terms proportional to $\ln ^{2} s$ are usually added in phenomenological fits. Even then the predictions [3, 4] are not completely satisfactory. In connection with the power-like preasymptotical behavior of $\sigma_{t}$ one could expect at present energies the faster than logarithmical shrinkage of the diffraction peak as well.

As a function of energy, the ratio $\rho_{0}$ increases from below, crosses zero and becomes positive at high energies. This is a general tendency for collisions of any initial particles. For $p p$-scattering, the prediction of (5) with values of $s$ scaled by $1 \mathrm{GeV}$ is still somewhat higher (about 0.177 ) than estimates from dispersion relations (0.14 in Refs [5, 6]) even at $7 \mathrm{TeV}$ while strongly overshoots them at ISR where $\pi / \ln s \approx 0.37$. No logarithmic decrease is seen in these predictions which, however, depend on behavior of the total cross section at higher energies. Moreover, the value 0.14 can only be reached according to (5) at the energy $75 \mathrm{TeV}$. Probably, at energies higher than 75 $\mathrm{TeV}$ the first signs of approach to asymptotics will become visible. No data about $\rho_{0}$ at the LHC energies exist yet.

Thus we can only hope that we are now in the preasymptotical regime, at best.

Surely, there is another more realistic possibility that the black disk model is too extreme and the gray fringe always exists. It opens the way to the nu- 
merous speculations with many new parameters about the particle shape and opacity (see, e.g., [7, 8, 9, 10, 11, 12, 14, 13, 15, 16, 17] which, nevertheless, are not as extreme as the black disk model.

However, let us study just the black disk model. There exists another parameter which can shed some light on the situation. This is the ratio

$$
Z=\frac{4 \pi B}{\sigma_{t}}
$$

which should be equal to $1 / 2$ for black disks. In the Table 1 we show how this ratio evolves with energy. All entries there except the last two are taken from Ref. [7] with simple recalculation $Z=1 / 4 Y$. The data at Tevatron and LHC energies are taken from Refs [18, 1]. All results are for $p p$-scattering except those at 546 and $1800 \mathrm{GeV}$ which are for $p \bar{p}$ processes. The accuracy of the numbers listed in the Table 1 can be very approximately estimated as \pm 0.1 from known error bars for the total cross sections and the slopes.

Table 1

$$
\begin{array}{lllllll}
\sqrt{s}, \mathrm{GeV}-2.704 .114 .746 .276 .98 & 7.6213 .862 .554618007000 \\
Z \quad-0.641 .021 .091 .261 .31 & 1.341 .451 .501 .201 .081 .00
\end{array}
$$

We see that $Z$ increases from low energy region to ISR energies and then drops down approaching 1 at LHC. Our interest in this ratio is related with the fact that the differential cross section of elastic scattering beyond the diffraction peak in the so-called Orear region is very sensitive to its value.

This region can be well fitted at different energies from rather low ones [19] to TOTEM data at LHC [20] by the very general expressions describing the cross section falloff with the dip-bump substructure derived in Refs [21, 22]. It was shown there that the unitarity relation outside the diffraction peak can be reduced to the integral equation for the imaginary part of the amplitude. No assumptions have been made other than the validity of experimental data in the diffraction peak. Using the shape of the amplitude in the diffraction cone

$$
A_{d}(p, \theta)=4 i p^{2} \sigma_{t} e^{-B p^{2} \theta^{2} / 2}\left(1-i \rho_{d}\right),
$$

one gets the unitarity equation written as

$$
\operatorname{Im} A(p, \theta)=\frac{p \sigma_{t}}{4 \pi \sqrt{2 \pi B}} \int_{-\infty}^{+\infty} d \theta_{1} e^{-B p^{2}\left(\theta-\theta_{1}\right)^{2} / 2}\left(1+\rho_{d} \rho_{l}\right) \operatorname{Im} A\left(p, \theta_{1}\right)+F(p, \theta) .
$$


The amplitude is denoted by $A . p \approx \sqrt{s} / 2$ and $\theta$ denote the momentum and the scattering angle in the center of mass system. $\rho_{i}$ 's take into account the real parts at the corresponding angles in the diffraction cone $(i=d)$ and at larger angles $(i=l)$. The integral term represents the two-particle intermediate states of the incoming particles. The function $F(p, \theta)$, called following Ref. 23] as the overlap function, represents the shadowing contribution of the inelastic processes to the elastic scattering amplitude.

Its analytic solution was obtained in a model-independent way with the assumptions that the role of the overlap function $F(p, \theta)$ is negligible outside the diffraction cone ${ }^{1}$ and the real parts may be replaced by their average values in the diffraction peak $\rho_{d}$ and outside it $\rho_{l}$, correspondingly. Let us stress once more that the Gaussian shape of the amplitude has been only used at rather small angles in accordance with experimental data. If it is ascribed to the Pomeron exchange, one is tempted to consider this solution as an effect of shadowing corrections due to multi-Pomeron graphs. Such corrections near the diffraction cone were considered, e.g., in Refs. [25, 26]. Even though their conclusions differ, the qualitative tendency of peak suppression is valid. The definite model summing multi-Pomeron graphs was considered in [27]. However, the oscillations over the exponentially damped regime happened to be too strong. The non-linear unitarity equation was solved by iterations assuming the Gaussian shape of the overlap function 22 but did not lead to quantitative agreement with experiment. That is ascribed to the ad hoc chosen shape of the overlap function which was recognized as unsatisfactory in earlier works (e.g., see [28, 29, 30, 31]). The solution of Eq. (8) does not depend on model assumptions.

Here we show only the leading term of the solution describing the exponential falloff with the angle $\theta$ (or $\sqrt{|t|}$ ) within the Orear regime which is most important for our purpose:

$$
\operatorname{Im} A(p, \theta)=C_{0}(p) \exp \left(-\sqrt{2 B \ln \frac{Z}{1+\rho_{d} \rho_{l}}} p \theta\right)
$$

The solution predicts the dependence on $p \theta \approx \sqrt{|t|}$ but not the dependence

\footnotetext{
${ }^{1}$ The results of the papers [20, 24] give strong support to this assumption. The overlap function has been calculated there directly from experimental data subtracting the elastic contribution $I_{2}$ from the lefthand side of the unitarity equation wthout model assumptions. It is extremely small outside the diffraction peak, and becomes even smaller at the LHC enargy compared to the lower ones.
} 
on the collision energy. Its validity may be checked by the direct substitution in Eq. (8). The values of the parameter $Z$ discussed above are crucial for the slope of the differential cross section in the Orear region. Another important parameter is the product of the average values of ratios of real to imaginary parts of the amplitude in the diffraction cone and in the Orear region. While the former ratio can be estimated from the experimental results on interference of Coulomb and nuclear amplitudes supported by calculations with the help of the dispersion relations, the latter one was never estimated from experimental data. Any information about it becomes valuable. That is why it is important that from the fit [20] at $7 \mathrm{TeV}$ it became possible for the first time to get its estimation from the experimentally measured slope in the Orear region. It happened to be surprisingly large in the absolute value $\left(\rho_{l} \approx-2\right)$. The high sensitivity of the expression (9) to this parameter is determined by the value of $Z$ being practically equal to 1 as seen in the Table 1 and, correspondingly, of $\ln Z$ very near 0 .

Once the expression for the imaginary part of the amplitude (9) outside the diffraction peak is known, it is possible to calculate the real part in the same region assuming the geometrical scaling [32] is valid. This has been done in [33]. It is shown that the real part changes its sign in accordance with the general theorem proven in [34] and becomes negative in the Orear region. Its absolute values happen, however, to be much smaller than those required by the fit of TOTEM data at $7 \mathrm{TeV}$. This problem has not been resolved yet.

Now, the question arises about what happens if the black disk limit of $Z=1 / 2$ will be approached at higher energies. If the exponential slope persists, the only solution would be to use even higher absolute values of $\rho_{l}$ (but negative!). However, this would show that there is no approach to black disks at all. This limit asks for $\rho_{d} \propto \ln ^{-1} s$ and, consequently, to $\rho_{l} \propto \ln s$ that seems too exotic possibility. If the product $\rho_{d} \rho_{l}$ tends to zero that looks more realistic, then the behavior of the differential cross section in the Orear region should become cardinally changed. In place of the exponentially decreasing falloff, the oscillatory behavior should appear. Now, the solution of the equation (8) looks as

$$
\operatorname{Im} A(p, \theta)=C_{0}(p) \cos \left(\sqrt{2 B \ln \frac{1+\rho_{d} \rho_{l}}{Z}} p \theta\right) .
$$

The imaginary part of the amplitude dominates and determines the shape of 
the differential cross section

$$
\frac{d \sigma}{d t}=\frac{C_{0}^{2}}{16 \pi s^{2}} \cos ^{2}\left(\sqrt{2 B \ln \frac{1+\rho_{d} \rho_{l}}{Z}|t|}\right) .
$$

The oscillatory behavior with periodically placed zeros is clearly seen.

In the black disk limit $Z=1 / 2 ; \rho_{i}=0 ; B=R^{2} / 4$. Thus

$$
\operatorname{Im} A(p, \theta)=C_{0}(p) \cos \left(\sqrt{0.5 R^{2} \ln 2|t|}\right)=C_{0}(p) \cos \left(\sqrt{\sigma_{t} \ln 2 / 4 \pi} p \theta\right) .
$$

The distance between oscillation zeros becomes very small and asymptotically tends to zero

$$
\Delta(\sqrt{|t|})=\frac{\pi}{\sqrt{2 B \ln \frac{1+\rho_{d} \rho_{l}}{Z}}} .
$$

It is hard to predict at what energies this transition from one regime to another will happen. The only guess I can propose is to rely on possible approach of the values of $\rho_{d} \approx \rho_{0}$ evaluated from dispersion relations to the black disk limit (51). As discussed above, it can happen at energies about 75 $\mathrm{TeV}$.

In some way, the conclusion about the undamped oscillations looks contradictory. Hardly the differential cross section will not decrease with $|t|$ at all. It is more probable that the factor $C_{0}^{2} / s^{2}$ is so strong that the regime (11) dies out very fast with energy increase. Then it becomes unobservable and the place of the Orear region is filled by the hard parton scattering. The exponential with $|t|^{1 / 2}$ falloff will be replaced by the power-like decrease.

Anyway we conclude that the approach to the black disk limit will induce drastic changes in the behavior of the differential cross section in the Orear region replacing the exponential falloff either by undamped oscillations in some energy interval or, that is more probable, by power-like behavior due to the hard parton scattering. It is possible that this feature will become the very first one noticeable if collision partners remind the black disks at extremely high energies.

\section{Acknowledgement}

This work was supported by the RFBR grant 12-02-91504-CERN-a and by the RAN-CERN program. 


\section{References}

[1] TOTEM Collaboration, G. Antchev et al., arXiv:1110.1385; 1110.1395.

[2] H. Cheng , J.K. Walker, T.T. Wu, Phys. Rev. D 9 (1974) 749.

[3] V.A. Okorokov, arXiv:0907.0951

[4] S.D. Campos, V.A. Okorokov, arXiv:1002.159

[5] I.M. Dremin, M.T. Nazirov, ZhETF Pis'ma 37 (1983) 163.

[6] M. Block, F. Halzen, arXiv:1102.3163.

[7] T.T. Chou, C.N. Yang, Phys. Lett. B 128 (1983) 457.

[8] C. Bourelly, J. Soffer, T.T. Wu, Eur. Phys. J. C 28 (2003) 97.

[9] P. Desgrolard, M. Giffon, E. Martynov, E. Predazzi, Eur. Phys. J. C 16 (2000) 499.

[10] M.M. Islam, R.J. Luddy, A.V. Prokudin, Phys. Lett. B 605 (2005) 115;

Int. J. Mod. Phys. A 21 (2006) 1.

[11] K. Golec-Biernat, M. Wüsthoff, Phys. Rev. D 60 (1999) 114023.

[12] V. Petrov, E. Predazzi, A. Prokudin, Eur. Phys. J. C 28 (2003) 525.

[13] B.Z. Kopeliovich, I.K. Potashnikova, B. Povh, E. Predazzi, Phys. Rev. D 63 (2001) 054001.

[14] M.M. Block, E.M. Gregores, F. Halzen, G. Pancheri, Phys. Rev. D 60 (1999) 054024.

[15] A. Kovner, U.A. Wiedemann, Phys. Rev. D 66 (2002) 034031.

[16] S.M. Troshin, N.E. Tyurin, arXiv:1111.4454.

[17] C. Merino, Yu.M. Shabelski, arXiv:1204.0769.

[18] N.A. Amos et al. Phys. Lett. B 247 (1990) 127.

[19] I.V. Andreev, I.M. Dremin, I.M. Gramenitskii, Nucl. Phys. 10 (1969) 137. 
[20] I.M. Dremin, V.A. Nechitailo, Phys. Rev. D 85 (2012) 074009.

[21] I.V. Andreev, I.M. Dremin, ZhETF Pis'ma 6 (1967) 810.

[22] I.V. Andreev, I.M. Dremin, Sov. J. Nucl. Phys. 8 (1968) 473.

[23] L. Van Hove, Nuovo Cimento 28 (1963) 798.

[24] I.V. Andreev, I.M. Dremin, D.N. Steinberg, Sov. J. Nucl. Phys. 11 (1970) 261.

[25] V.A. Khoze, A.D. Martin, M.G. Ryskin, Eur. Phys. J. 18 (2000) 167.

[26] E. Gotsman, E. Levin, U. Maor, Phys. Lett. B 353 (1995) 526.

[27] A.A. Anselm, I.T. Dyatlov, Phys. Lett. B 24 (1967) 479; Yad. Fiz. 6 (1967) 591 (Sov. J. Nucl. Phys. 6 (1968) 430).

[28] H. Fukuda, C. Iso, Nuovo Cim. 43 (1966) 43.

[29] K. Zalewski, L. Van Hove, Nuovo Cim. 46 (1966) 806.

[30] L. Michejda, Nucl. Phys. B 4 (1967) 113.

[31] M. Giffon, Y. Hama, E. Predazzi, Z. Phys. C 19 (1983) 341.

[32] J. Dias de Deus, Nucl. Phys. B 59 (1973) 231.

[33] I.M. Dremin, arXiv:1204.1914.

[34] A. Martin, Phys. Lett. B 404 (1997) 137. 